\title{
Capítulo I 3 \\ Oferta de espacios informativos en la radio lagunera
}

\author{
Blanca Chong López* \\ Alfredo Morales Pérez ${ }^{* *}$ \\ Universidad Autónoma de Coahuila, Unidad Torreón
}

Pese al importante desarrollo de otros medios, la radio sigue teniendo una amplia presencia en la cotidianeidad de gran parte de los mexicanos. Entre los géneros que incluye el medio radiofónico, a partir de los noventa y respondiendo a las transformaciones de la sociedad mexicana que demanda una mayor apertura y pluralidad, los espacios informativos se convirtieron en un elemento central de su actividad.

En este trabajo se tiene como objetivo un acercamiento a los programas informativos que se ofrecen en el cuadrante radiofónico de la Comarca Lagunera. Aunque no es sencillo establecer lo que es calidad, hemos considerado una definición desde la perspectiva de las audiencias, como base para el análisis de lo que se realiza en cuanto al trabajo informativo en las emisoras regionales. El resultado es seguramente similar a lo que ocurre en otras regiones del país: predominio de programas transmitidos desde la Ciudad de México y en general una producción que no ofrece pluralidad ni profundidad en los contenidos, en las emisiones locales.

In spite of the important development of other media, radio is still having a wide dayly presence in grand part of Mexicans. Between the genders that

\footnotetext{
* Doctora en Ciencias de la Comunicación por la Universidad de La Habana. Profesora de tiempo completo en la Facultad de Ciencias Políticas y Sociales de la Universidad Autónoma de Coahuila.

** Lic. en Sociología por la Universidad Autónoma de Coahuila, con estudios de Maestría en Comunicación en la Universidad Iberoamericana. Profesor de tiempo completo en la Facultad de Ciencias Políticas y Sociales de la Universidad Autónoma de Coahuila.
} 
includes the radiophonic media, from the ninetys (90's) on and answering to the transformations of the mexican society wich demands a major opening and plurality, the informative spaces have turned in to a central element of its activity.

This investigation has as an objective an approachment to the informative programs offered in the Comarca Lagunera's radiophonic quadrant. Although it's not simple to establish what is quality, we have considered a definition from the audience's perspective, as a base for the analysis of what is done with regard to the informative work in regional radio stations. The result is surely similar to what occurs in other country regions: the predominance of programs transmitted from Ciudad de México and in general a production that doesn't offer plurality nor deepness in contents, in local emissions.

\section{INTRODUCCIÓN}

Después de haber sido desplazado en buena medida por el auge de la televisión a fines de los setenta, pero especialmente en los ochenta, se dio un resurgimiento del medio radiofónico.

A partir de los últimos ańos de los ochenta la radio mexicana tuvo importantes cambios en su programación y contenidos, pero quizás el más notable fue la apertura a la expresión de los distintos actores políticos y sociales, en consonancia con las transformaciones que a partir de entonces vive nuestro país, las cuales obligaron a la radio a modificar su perfil, a darle mayores espacios de expresión a las audiencias, a superar esquemas ya agotados como la simple transmisión de información y la difusión de declaraciones de políticos. En ese sentido, los espacios informativos en la radio se convirtieron en un género fundamental de su actividad, aunque en muchas emisoras se siguió privilegiando el entretenimiento.

Para Sosa (1999), "el "boom" de los noticiarios radiofónicos, se explica no sólo por una razón financiera y de competencia frente a otros medios, sino también como consecuencia de un país que políticamente ha cambiado mucho y que por diferentes mecanismos de presión social, ha ensanchado los márgenes de la libertad de expresión y el derecho a la información a través de la radio. Aunado a este factor, se encuentra la necesidad de los propios radiodifusores de tener, en sus propios medios, canales de expresión para la defensa de sus intereses de grupo."

La radio comercial en México ha retomado las necesidades de información de los distintos sectores sociales, creando un mayor número de programas 
orientados al análisis y comentario de la noticia, dejando cada vez menos la discusión de los problemas que aquejan al país en manos de los funcionarios gubernamentales y permitiendo la posibilidad de escuchar voces que cuestionan el sistema político. (Gómez, 1992).

El trabajo que se presenta es un primer acercamiento descriptivo a la radio de la comarca lagunera para tratar de identificar los espacios informativos que ofrece, como parte del proyecto de investigación en red "Espacios de calidad en los medios electrónicos regionales", coordinado por Fátima Fernández.

Los cuestionamientos a los que se trató de dar respuesta fueron: ¿Cuál es la oferta de espacios informativos en las emisoras radiofónicas regionales?, ¿Qué características ofrecen los programas informativos en el cuadrante radiofónico de la región?, ¿Qué peso tienen los programas informativos transmitidos desde la Ciudad de México en las emisoras regionales?

\section{¿Cómo DEFINIR LA CALIDAD DE LOS PROGRAMAS?}

Como señala Fernández (2005), "cualquier definición de calidad implica una apropiación del término realizada desde una particular ubicación social”. Se trata de un concepto abierto que logra significado sólo al entrar en relación con otros conceptos.

Distintos estudiosos y grupos de trabajo han tratado de definir los elementos que deberían contener los programas de radio o televisión para poder ser considerados como de calidad. Se trata de visiones que en algunos casos generan problemas al tratar de aplicar sus criterios en el análisis de la programación, por ejemplo el hecho de que se considere la población como un bloque homogéneo, y no se tome en cuenta las diferencias culturales, ${ }^{1} \mathrm{o}$ bien por la imposibilidad de integrar la visión empresarial, periodística y social sobre aspectos subjetivos como la axiología. ${ }^{2}$

En el caso de los programas informativos hemos considerado pertinente retomar la propuesta que hace el Consejo Mundial de Radio y Televisión, organismo que en 2001 creó un estándar para la calidad de la industria audiovisual, quien a través de una de sus representantes expresa que existen por lo menos

${ }^{1}$ Esto ocurre en la visión del emisor a nombre de la sociedad, como en el caso de la Asociación "A favor de lo mejor".

${ }^{2}$ Lo que se observa en la propuesta de la Fundación Nuevo Periodismo Iberoamericano, cit. en Fernández (2005). 
tres sectores que pueden definir de manera diferente el problema de la calidad en los medios audiovisuales: los responsables de los medios, los creadores de los productos y las audiencias. Desde estas últimas, a las que denomina también perspectiva ciudadana, la calidad debe distinguirse de la popularidad y buscar: pluralismo, profundidad en los contenidos, diversidad en la programación, protagonismo social (Miralles, 2005).

Un aspecto importante a considerar en la calidad de los programas informativos es la posibilidad de que las audiencias tengan voz en ellos. Los noticiarios pueden ser espacios importantes para la expresión de inquietudes políticas y sociales, donde se vuelve "pública" la inconformidad ciudadana, lo que permite crear un espacio de interacción entre la población y los gobernantes (Winocur, 2000). La gente llama por diversos motivos: solicitar información, realizar denuncias por abusos de autoridad, reportar fallas en los servicios públicos, opinar sobre distintos temas de su interés.

Al tratar de conocer la oferta de espacios de calidad en la programación radiofónica no podemos dejar de lado los criterios bajo los que operan los medios comerciales, los cuales para asegurar el mayor número de audiencias reducen al mínimo los riesgos, reproduciendo fórmulas que se parezcan lo más posible a lo que ya está probado y limitando las innovaciones, pues "el contexto determinante para la producción es siempre el de su mercado. Para tratar de ampliar al máximo dicho mercado, los productores deben inclinarse a los valores primordiales más ampliamente legitimados y rechazar la voz disidente o la objeción incompatible con un mito dominante" (Murdock \& Golding, 1986, p. 53).

LA RADIO, UN MEDIO VIGENTE

En la era de Internet, la radio abierta tradicional sigue cumpliendo una importante función social, cultural y económica. Los beneficios que ofrece la radio son difícilmente sustituibles por una computadora, más aún considerando que gran parte de la población no tiene acceso a ella.

La radio es un medio que durante décadas ha permanecido cercano a la mayoría de los mexicanos de todos los sectores sociales, pero de manera particular a los de menores recursos económicos.

Pese al desarrollo de otros medios, principalmente la televisión, la radio continúa presente en la vida cotidiana de miles de personas para quienes representa la única posibilidad de comunicación más allá de sus localidades; para 
muchos otros, la radio es la "compañía" en el desarrollo de las actividades más diversas, se integra a la vida familiar y laboral.

Por tratarse de un pueblo donde la tradición oral es uno de sus rasgos culturales relevantes, indudablemente esa gran presencia de la radio se debe a que este medio tiene un uso social y cultural importante. El hecho de que en la mayoría de los hogares mexicanos exista por lo menos un aparato de radio, sólo puede explicarse a partir de lo que representa en su vida cotidiana.

Movilidad en la recepción, gratuidad del servicio, bajo costo de los receptores, bajo costo en su producción y distribución, su contribución a la cohesión social, así como a la educación formal e informal, a la preservación de la cultura tanto a nivel nacional, regional y local, son factores que hacen imprescindible seguir impulsando el desarrollo de la radio.

En relación a las características técnicas de la radio, Luis Bassets anota que: "frente a la dominación y manipulación de los grandes medios, frente a la complejidad de las grandes cadenas de televisión, a los altísimos costos de tecnologías duras, sólo cabe oponer la modesta, pero eficaz e incordiante alternativa de los pequeños y sencillos medios que son las radios locales, baratas y accesibles a todos, escurridizas al poder y susceptibles de acciones eficaces y puntuales en todos los terrenos: político, naturalmente, pero también cultural, sexual y familiar". (Rebeil, 1988, p. 25).

Algunos rasgos del medio que explican su importancia para amplios sectores de la sociedad, los señala Cristina Romo: "La radio es el medio ideal para hablar personalmente, para dirigirse a pequeños grupos, para poner en comunicación a grupos afines. También para informar de manera inmediata; proporcionar elementos de formación de la opinión pública; poner en comunicación a poblaciones aisladas, facilitar la movilización social y, también, para dar a conocer las bondades de productos y servicios, además de las novedades en el terreno de la música”. (Romo, 1996, p. 29).

Una característica que distingue a la radio y la televisión es que se trata de medios de primera necesidad para informarse y orientarse en la vida cotidiana, para obtener diversión y temas para compartir con los demás. En cuanto a información, la radio tiene la ventaja de la inmediatez, el poder emitir comunicados periódicamente, facilitar una mayor concentración en el mensaje, poder realizar otras actividades en forma simultánea. (Prieto, 1996, p. 14)

La utilización que hacen de la radio los oyentes es distinta según la situación. Para Claude Collín, "la radio es o podría ser, el medio de la confidencia, el lugar de la comunicación entre individuos, una manera de romper la soledad 
del hombre moderno, de brindarle el sentimiento de la relación individual que no encuentra en la vida cotidiana" (Collin, 1983, p. 55). Pero si en nuestra sociedad por lo general la radio se escucha en soledad, existen situaciones en las que la radio se escucha colectivamente, y el hecho de escuchar la radio brinda un sentimiento de pertenencia a una colectividad.

Además de sus características técnicas la vigencia de la radio se explica por su capacidad para ser comprendida por públicos heterogéneos, que no requieren de gran conocimiento para entender sus mensajes, no se requiere saber leer. La radio puede además ser utilizada con fines de educación, concientización y organización, particularmente entre los sectores populares.

La radio en el siglo XXI es un medio de comunicación vigente, frente a avanzadas tecnologías de comunicación que indudablemente representan un gran atractivo para muchas personas, lo cual podría parecer contradictorio. Sin embargo, son las características del medio, que ya se han enumerado, las que le permiten mantener una importante presencia dentro de la sociedad.

\section{MÉTOdo}

Para conocer la oferta de espacios informativos en la radio de la Comarca Lagunera se revisaron las páginas web de las empresas radiofónicas de la región, información que fue complementada con entrevistas a funcionarios de cada uno de los grupos, en las que además se trató de obtener mayores datos sobre los orígenes y desarrollo de las empresas.

La descripción de los programas informativos producidos localmente se realizó sintonizando cada uno de ellos, abarcando la totalidad de las emisoras del cuadrante regional. Esta actividad fue desarrollada durante el mes de julio de 2005.

ESPACIOS INFORMATIVOS EN LAS EMISORAS RADIOFÓNICAS DE LA Comarca Lagunera

La actividad radiofónica en La Laguna ${ }^{3}$ la desarrollan cinco grupos y una emisora de carácter cultural, que en total cuentan con 22 estaciones. En esta parte del

${ }^{3}$ La Comarca Lagunera, región ubicada en el centro-norte de México, está conformada por parte de los Estados de Coahuila y Durango. La Laguna, como también es conocida esta región, está integrada por 13 municipios, 8 del Estado de Durango y 5 del Estado de Coahuila. 
trabajo se describen brevemente los antecedentes de cada uno de los grupos, así como de la emisora cultural, y los espacios informativos que ofrecen.

\section{Radio Torreón}

En 1991 inició operaciones la primera y única radiodifusora cultural de la región, XHTOR "Radio Torreón", ubicada en el 96.3 FM, con un perfil inicial de repetidora de Radio Educación y parte de producción local, a cargo de Arte y Cultura del Municipio. Poco a poco fue modificando la relación entre programas producidos directamente y los apoyos de otras instituciones.

Radio Torreón es una emisora cultural y educativa permisionada, que trabaja con recursos del municipio de Torreón. Actualmente en el desarrollo de su actividad incluye programas de todo tipo: de difusión, con corte cultural y científico; cápsulas a lo largo de la programación, en las que se informa sobre las actividades del Ayuntamiento; educativos, donde participan instituciones de nivel medio superior y superior de la región. Se ofrecen espacios informativos que se refieren únicamente a los trabajos de la administración municipal: Vive el cambio, que se transmite de lunes a viernes de 14:00 a 14:30 hs. y Guillermo Anaya cerca de ti, los martes, de 8:00 a 9:00 hs., que busca establecer comunicación directa entre el Presidente Municipal, los funcionarios del Ayuntamiento y los ciudadanos.

\section{Corporación Multimundo}

Multimundo es el grupo de radio más joven en La Laguna. Inició sus operaciones en 1994, teniendo su sede en la ciudad de Querétaro. En Torreón cuenta con dos emisoras, una de ellas, "Radio Fórmula", que se localiza en la frecuencia 740 AM con una programación de radio hablada, transmitida casi en su totalidad desde la Ciudad de México. En esta radiodifusora se tienen nueve programas informativos que se transmiten desde la capital del país y sólo un espacio informativo local, Sin fórmula, que se difunde en dos horarios, de 13:00 a 13:30 hs. y a las 19:00 hs. con una hora de duración, en el que se brinda información internacional, nacional, regional y deportiva. Una característica de este noticiario es el estilo informal con que comentan las noticias los conductores, y la invitación que hacen a la audiencia para que participe.

En la otra emisora del grupo, 100.3 Kiss FM se transmite desde la Ciudad de México el noticiario Imagen Informativa, con una duración de casi cuatro horas diarias, y un espacio informativo producido localmente Digestivo, que realiza el mismo equipo que hace Sin fórmula, mezcla de música con noticias locales, centradas en temas políticos. Este programa dura una hora. 


\section{Grupo Radio Estéreo Mayrán, (GREM)}

Fundado en 1985, aunque sus tres emisoras existían con anterioridad, es conocido como un grupo netamente "lagunero", por ser la única organización radiofónica que pertenece a empresarios de la región. En este grupo se tuvieron las dos primeras estaciones de FM en la Comarca Lagunera. En cuanto a su perfil, GREM ha sido pionero en el desarrollo de la radio hablada en la región. Actualmente las emisoras del grupo son XETC 880 AM, “Tu música”, XHPE 97.1 FM "Estéreo Gallito"y XHMP 95.5 "Sentidos".

Dentro de los espacios informativos que cuenta este grupo, el programa Contextos, difunde una síntesis informativa internacional, nacional, regional y deportiva. Se centra en el análisis de la información regional más relevante y se tienen invitados en diferentes temas. Se cuenta con una sección denominada "la voz de los protagonistas" en la que el titular del noticiario presenta las declaraciones de personas que son parte del hecho noticioso. En algunos momentos del noticiario se leen los comentarios de los radioescuchas. Se transmite durante tres horas, de 7:00 a 10:00 hrs., de lunes a viernes, en una de las estaciones del grupo, $880 \mathrm{AM}$.

El programa Actualidades GREM, es uno de los espacios informativos con mayor audiencia a nivel regional, se transmite de 13:00 a 14:00 hrs., de lunes a sábado en las tres estaciones del grupo. En este programa se da la opción a la ciudadanía para que participe con opiniones y quejas sobre servicios públicos que el gobierno debe proveer. Los conductores frecuentemente orientan sobre la manera en que se pueden resolver los problemas, y en ocasiones se cuenta con la presencia de algún funcionario público que responde directamente los cuestionamientos. Durante los lapsos en que no hay llamadas se difunden notas locales.

El Correo de las Siete es un programa que se transmite por las tardes, a las 19:00 hrs., de lunes a viernes, con una duración de media hora, a través de las tres emisoras del grupo. Centrado en la información regional, al final de este espacio se informa brevemente de los acontecimientos más relevantes en lo internacional y lo nacional, así como en el ámbito deportivo. Al terminar la primera sección de noticias se incluye un editorial sobre algún tema de interés en la región.

A tiempo es un noticiario matutino que se difunde simultáneamente en dos emisoras del grupo, 97.1 FM y 95.5 Sentidos. de 7:30 a 7:50 hrs. con lo más relevante de la información local, nacional, internacional, financiera y deportiva. Al finalizar se incluye una sección llamada "la otra información", en la que se leen dos noticias curiosas que hayan ocurrido en el mundo. 
En las tres radiodifusoras del grupo se transmiten de lunes a viernes, en diferentes horarios, pequeños espacios informativos denominados GREM en la Información, donde se difunden los acontecimientos más recientes. Se trata de cápsulas de información regional que tienen una duración de dos minutos.

En esta empresa radiofónica desde finales de la década de los noventa no se transmiten programas informativos desde la Ciudad de México.

\section{Multimedios Estrellas de Oro}

Fundado en 1992, este grupo cuenta con dos emisoras, XHCTO "Stereo Hits" en el 92.1 FM y XHTRR "La caliente" que se localiza en el 92.3 FM. Pertenece a la empresa Multimedios Estrellas de Oro, de Monterrey, N. L., que en la ciudad de Torreón posee además una televisora y un periódico. En ambas radiodifusoras se difunde de manera simultánea un noticiario en tres horarios, de lunes a viernes, con 12 minutos de duración, en el que se ofrece información internacional, nacional, local y deportiva. En estas emisoras no se transmiten espacios informativos de la Ciudad de México.

\section{Organización Impulsora de Radio (OIR Laguna).}

Integrado por cinco emisoras, este grupo, constituido inicialmente como Radio Laguna, fue fundado hace 39 años, con estaciones que hasta ese momento funcionaban de manera independiente. La primera de las estaciones de OIR Laguna fue XETB, "Radio Laguna" ubicada en el 1350 AM, que inició sus operaciones en 1931, en los primeros años de la radiodifusión en nuestro país. Otras radiodifusoras de este grupo son XETOR "Radio Ranchito" 670 AM, XEWN “El Fonógrafo" 1270 AM, XHRCA "Stereo Joya” 102.7 FM, XHLZ, "La Z”, 103.5 FM.

La mayor parte de la programación de las emisoras de este grupo está constituida por música de todo tipo, desde romántica hasta grupera; cada emisora se especializa en alguno de ellos.

El espacio informativo de producción local más importante con que cuenta esta empresa es OIR Noticias, que se transmite en "Radio Ranchito" en horario matutino, con una hora de duración de lunes a viernes, y en "Radio Ranchito" y "Radio Laguna”, al mediodía, igualmente con una hora de duración, de lunes a viernes. Aunque se aborda información internacional y nacional, en este programa se hace énfasis en lo regional, y se da oportunidad al público de expresar sus quejas, principalmente contra funcionarios públicos. Se tiene además un espacio de media hora, Resumen OIR Noticias, que se transmite por la tarde en tres de las cinco emisoras del grupo. 
En las estaciones de esta empresa radiofónica se transmiten cuatro programas informativos desde la Ciudad de México.

\section{Organización Radiofónica del Centro. Radiorama de La Laguna}

El de Radiorama es el caso más representativo de centralización de la radio a nivel regional. Este consorcio maneja ocho de las estaciones locales. Una de las emisoras del grupo, la XEBP, "La Bonita" 1450 AM, es la segunda más antigua de la Comarca Lagunera, inició sus operaciones en 1939. En las estaciones de este grupo la programación se compone fundamentalmente de espacios musicales.

Un espacio informativo que ofrece esta empresa es el Noticiero NN Nuestras Noticias, que se transmite de 13:00 a 14:00 hs. de manera simultánea por tres emisoras del grupo: XEGZ "W Radio", en el 790 AM, XEVK "La K Buena" en el 1010 AM y XEDN "La Mexicana", en el 600 AM. En "W Radio" se ofrece también el noticiario local Contrapunto, que se transmite de 18:00 a 19:00 hs. con información regional, nacional e internacional, pero se hace énfasis en lo local.

En XEYD "La grande de Madero", 1410 AM, se difunde el programa Tribuna libre, que se presenta como un espacio abierto para que la población exprese la problemática en la que está inmersa. No se incluye notas periodísticas, y sus conductores permiten la entrada de llamadas telefónicas para enviar saludos al auditorio.

En las radiodifusoras de esta empresa se transmiten desde la Ciudad de México cinco programas informativos diariamente.

A MANERA DE CONCLUSIÓN

En suma, el cuadrante radiofónico regional ofrece 18 programas informativos que se transmiten desde la Ciudad de México, y 13 producidos localmente, cinco de ellos en Grupo Radio Estéreo Mayrán, GREM. En los noticiarios locales la diferencia entre unos y otros radica sobre todo en el estilo de los conductores, la profundidad con que se aborda la información, y las posibilidades que se brindan al público radioescucha para que exprese sus opiniones.

El predominio de programas transmitidos desde la capital se explica a partir de criterios económicos. Resulta más redituable a las empresas locales pagar la repetición que asumir los costos de una producción con las características de las realizadas en la Ciudad de México. 
¿Es posible hablar de calidad en los programas informativos que se producen en la radio regional, considerando los criterios expuestos al inicio de este trabajo?. Consideramos que existe un esfuerzo serio en el grupo lagunero, GREM, por ofrecer a la audiencia espacios plurales, en los que se aborde con profundidad la información. En un estudio de audiencias realizado en 1997, al preguntar sobre los programas informativos que escuchaban, el mayor porcentaje tanto en el medio urbano como rural del municipio de Torreón lo obtuvo precisamente un noticiario de este grupo, que aún se mantiene al aire, El correo de las siete. (Chong, 1998). Creemos así mismo que en los espacios informativos locales del grupo Multimundo se trata de innovar, con un estilo que puede resultar atractivo a los radioescuchas. En lo que se refiere a la posibilidad de que los radioescuchas se expresen a través de estos programas, sólo dos de ellos lo permiten, a pesar de la importancia que tiene para la interacción entre los ciudadanos y las autoridades. Llama la atención el hecho de que la única emisora pública de la región no transmita programas informativos, fuera de lo que tiene que ver con el Ayuntamiento de Torreón, del cual depende.

Con seis escuelas que en la región ofrecen la licenciatura en Comunicación, sería importante que en ellas se impulsara una revalorización de este medio, a fin de que las nuevas generaciones de comunicadores se interesaran en desarrollar mejores propuestas para la radio del futuro. Es necesario contar con productores que conciban a la radio como espacio expresivo de la sociedad, pues como señala Sosa (1999), "Para cumplir cabalmente su labor social y continuar como una alternativa seria de difusión informativa, la radio aún tiene por delante algunos retos que van desde una mayor apertura de espacios a las distintas corrientes políticas hasta recuperación de los públicos que la televisión le ha arrebatado en los últimos ańos".

La radiodifusión en nuestro país es factor primordial en la construcción de sentido. La naturaleza de una red como la de la radio no se limita a proporcionar esparcimiento y diversión, incide en la generación de otro tipo de vínculos culturales y sociológicos.

\section{Bibliografía}

Carabaza, J. y Ewald, I. (1995). Historia de los medios de comunicación en Coahuila. México: UA de C.

Collin, C. (Radiopoder). (1983). La radio como instrumento de participación social y politica. México: Folios Ediciones. 
Chong, B. (1998). La radio en La Laguna. Un estudio de audiencias. Tesis de Maestría. México: UIA.

Fernández, F. (2005). ¿Cómo definimos calidad?. México: Manuscrito no publicado. Varios. (1991). La radio mexicana. Centro y regiones. México: Juan Pablos.

Gómez, H. (1992, enero-agosto). En búsqueda de la audiencia radiofónica, Comunicación y Sociedad, pp. 14-15.

Prieto, F. (1995-1996, noviembre-enero). Función social de la radio, Revista Mexicana de Comunicación, No. 42.

Miralles, A. (Cit. por Férnandez) (2005). ¿Cómo definimos Calidad?. Artículo: La ciudadanía en la calidad de los medios, Democracia y Medios Públicos. Memorias del Primer Congreso Internacional organizado por la Red de Radiodifusoras y Televisoras Educativas y Culturales de México. Manuscrito no publicado.

Murdock, G. \& Golding, P. (1986). Capitalismo, comunicaciones y relaciones de clase, Sociedad y comunicación de masas. México: F.C.E.

Rebeil, M. A., et. al. (1989). Perfiles del Cuadrante: Experiencias de la radio. México: Trillas.

Romo, C. (1996, primavera). La radio, vigente en el umbral del Siglo XXI, $L a$ Radio, No. 1.

Sosa G. (2003, septiembre-octubre). Crisol de expresiones, Revista Mexicana de Comunicación, No. 83.

Varios. (2004). La radio en la era de Internet, Radio World América Latina, Vol. 28, No. 1.

Varios. (1999, julio-agosto). Situación actual y retos de los noticiarios radiofónicos en la Ciudad de México, Revista Universidad Nacional, UNAM. Recuperado el 30 de abril, 2006, de www.radiomexicana.com

Winocur, R. (2000). La participación en la radio, una posibilidad negociada de ampliación del espacio público, Dia-logos de la Comunicación, No. 58. 